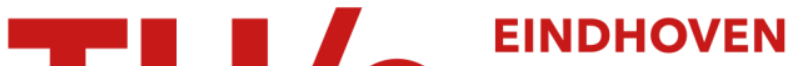 \\ UNIVERSITY OF \\ TECHNOLOGY
}

\section{Hot cracks in rubber: origin of the giant toughness of rubber- like materials}

Citation for published version (APA):

Carbone, G., \& Persson, B. N. J. (2005). Hot cracks in rubber: origin of the giant toughness of rubber-like materials. Physical Review Letters, 95(11), 114301-1/4. [114301].

https://doi.org/10.1103/PhysRevLett.95.114301

DOI:

10.1103/PhysRevLett.95.114301

Document status and date:

Published: 01/01/2005

\section{Document Version:}

Publisher's PDF, also known as Version of Record (includes final page, issue and volume numbers)

\section{Please check the document version of this publication:}

- A submitted manuscript is the version of the article upon submission and before peer-review. There can be important differences between the submitted version and the official published version of record. People interested in the research are advised to contact the author for the final version of the publication, or visit the $\mathrm{DOI}$ to the publisher's website.

- The final author version and the galley proof are versions of the publication after peer review.

- The final published version features the final layout of the paper including the volume, issue and page numbers.

Link to publication

\section{General rights}

Copyright and moral rights for the publications made accessible in the public portal are retained by the authors and/or other copyright owners and it is a condition of accessing publications that users recognise and abide by the legal requirements associated with these rights.

- Users may download and print one copy of any publication from the public portal for the purpose of private study or research.

- You may not further distribute the material or use it for any profit-making activity or commercial gain

- You may freely distribute the URL identifying the publication in the public portal.

If the publication is distributed under the terms of Article 25fa of the Dutch Copyright Act, indicated by the "Taverne" license above, please follow below link for the End User Agreement:

www.tue.nl/taverne

Take down policy

If you believe that this document breaches copyright please contact us at:

openaccess@tue.nl

providing details and we will investigate your claim. 


\title{
Hot Cracks in Rubber: Origin of the Giant Toughness of Rubberlike Materials
}

\author{
G. Carbone $\mathrm{e}^{1,2,3}$ and B. N. J. Persson ${ }^{2}$ \\ ${ }^{1}$ DIMeG-Politecnico di Bari, Via le Japigia 182, 70126 Bari, Italy \\ ${ }^{2}$ IFF Forschungszentrum Jülich, 52425 Jülich, Germany \\ ${ }^{3}$ CEMeC-Politecnico di Bari, Via Re David 200, 70125 Bari, Italy
}

(Received 29 March 2005; published 8 September 2005)

\begin{abstract}
We study crack propagation in rubberlike materials and show that the nonuniform temperature distribution which occurs in the vicinity of the crack tip has a profound influence on the crack propagation, and may strongly enhance the crack propagation energy $G(v)$ for high crack velocities $v$. At very low crack-tip velocities, the heat produced at the crack tip can diffuse away, but already at moderate crack-tip velocities a very large temperature increase occurs close to the crack tip resulting in a "hot-crack" propagation regime. The transition between the low-speed regime and the hot-crack regime is very abrupt and may result in unstable crack motion, e.g., stick-slip motion or catastrophic failure.
\end{abstract}

DOI: 10.1103/PhysRevLett.95.114301

The invention of vulcanization by Charles Goodyear in 1839 represents one of the most important discoveries in materials science. Natural rubber is, at room temperature, a sticky substance (high-viscosity liquid) of limited technological importance. After cross linking of the long hydrocarbon chains it forms a soft elastic solid with remarkable elastic, frictional, and toughness properties, which forms the basis for most practical applications involving rubber, e.g., for tires, wiper blades, sealings, and conveyor belts [1].

Rubber has one of the highest toughnesses of all known materials. The toughness reflects the high energy $G$ necessary to propagate cracks $[2-4]$. Thus, the crack propagation energy $G$ at crack velocities of order $\sim 1 \mathrm{~cm} / \mathrm{s}$ may be a million times higher than the surface energy of rubber. This shows that the classical Griffith theory [5], where $G$ is identified with the surface energy $2 \gamma$ of the created surfaces, is not directly valid for rubberlike materials. The strong enhancement of $G$ is mainly due to the viscoelastic energy dissipation in front of the crack tip, which occurs even at very low crack-tip velocities, e.g., $1 \mu \mathrm{m} / \mathrm{s}$. In this Letter we will show that the heating of the rubber close to the crack tip enhances this viscoelastic energy dissipation, and may result in crack propagation instabilities.

The strength of adhesion and cohesion of elastomers can be characterized by the amount of energy $G$ required to advance the crack tip by one unit area. Experiments have shown that $G$ depends on the crack-tip velocity $v$ and on the temperature $T$ and that [2-4] $G(v, T)=G_{0}[1+$ $f(v, T)$ ], where $f \rightarrow 0$ as $v \rightarrow 0$. Here $f(v, T)$ describes the bulk viscoelastic energy dissipation well ahead of the crack tip, where the stress and strain are so small that linear viscoelasticity theory should be a good approximation. Thus, this term is determined by the viscoelastic modulus $E(\omega, T)$ of the rubber. The factor $G_{0}=2 \gamma_{0}$ is due to the bond breaking at the crack tip (in the so called crack-tip process zone), which involves highly nonlinear processes [e.g., cavity formation, stringing, chain pullout (for polymers), and bond breaking]. This factor cannot be calcu-
PACS numbers: 46.50.+a, 62.20.Fe, 62.20.Mk

lated theoretically at present, and is taken as an experimental input in the theory presented below. The strongest velocity and temperature dependence of $G(v, T)$ is derived from the factor $f(v, T)$, which at high crack-tip velocities may enhance $G$ by a factor $10^{3}$ or more.

Consider a crack loaded in tension (mode I) in a viscoelastic solid. We calculate the crack propagation energy $G(v)$, which is an intrinsic material quantity that does not depend on the geometry of the sample, or how the system is loaded: in the following we focus on the simplest case of plane stress [6]. For plain stress, the crack propagation energy $G$ is given by [7] $G=K^{2} / E$, where $E$ is the elastic modulus. The stress intensity factor $K$ is proportional to the applied stress. Let us first calculate the bulk energy dissipation per unit time and unit length of the crack line $P$ for the general case of a crack propagating with velocity $\mathbf{v}$ in a linear viscoelastic solid. We have

$$
P=\int d^{2} x \dot{\epsilon}_{i j} \sigma_{i j}
$$

where $\sigma_{i j}$ is the stress tensor and $\dot{\epsilon}_{i j}$ the strain rate tensor. In continuum mechanics, for a homogeneous material, the general form of the stress in the vicinity of a crack tip is independent of the detailed relation between the stress and strain (i.e., also valid for a viscoelastic material), and takes the universal form [7]

$$
\sigma_{i j}(\mathbf{x}, t) \approx \frac{K}{(2 \pi|\mathbf{x}-\mathbf{v} t|)^{1 / 2}} f_{i j}(\phi) .
$$

The tensorial aspect of the stress tensor enters via the universal function $f_{i j}(\phi)$ that depends on the polar angle $\phi$ in the $x y$ plane.

Now, let us consider the energy conservation condition relevant to the crack propagation. In the present case, the elastic energy stored in the solid in front of the crack tip is dissipated at the crack tip. The flow of elastic energy into the crack is given by $G v$, which must equal the fracture energy $G_{0} v$ plus the bulk viscoelastic dissipation $P$ given 
by (1): $G v=G_{0} v+P$. Substituting (2) into (1) and using $K^{2}=G E_{0}$ where $E_{0}=E\left(0, T_{0}\right)$ (where $T_{0}$ is the background temperature) gives [6]

$$
\begin{aligned}
\left(\frac{G}{G_{0}}\right)^{-1}= & 1-\frac{2}{\pi} \int_{0}^{1} d x x \\
& \times \int_{x}^{1} d y y^{-2}\left(y^{2}-x^{2}\right)^{-1 / 2} \operatorname{Im} \frac{E_{0}}{E\left(x \omega_{c}, T_{y}\right)} .
\end{aligned}
$$

In (3) we have introduced the large cutoff wave vector $q_{c}=2 \pi / a$, and the corresponding large cutoff frequency $\omega_{c}=2 \pi v / a$, where $a$ is taken equal to the radius of the crack tip. $T_{q}$ is the temperature in the spatial region in front of the crack tip probed by the $q$-wave number contribution to the integral (3), and defined as $T_{q}=\int d^{2} x \Phi_{q}(\mathbf{x}) T(\mathbf{x})$ with $\Phi_{q}(\mathbf{x})=(q / 2 \pi) \delta(r-1 / q)$. We have defined $q=$ $y q_{c}$ and $\omega=x \omega_{c}$, and $T_{y}$ stands for $T_{q}$ with $q=y q_{c}$.

The radius of curvature of the crack tip $a$ can be calculated by considering that, in order for the crack to propagate, the stress at the crack tip must be equal to some characteristic (material dependent) yield stress $\sigma_{c}$. Using (2), with $\sigma$ replaced by $\sigma_{c}$ and $|\mathbf{x}-\mathbf{v} t|$ by $a$, leads to the relation $2 \pi \sigma_{c}^{2} a=K^{2}$. Substituting $K^{2}=G E_{0}=2 \gamma_{\mathrm{eff}} E_{0}$ this equation gives $\sigma_{c}^{2} a=E_{0} \gamma_{\mathrm{eff}} / \pi$, which shows that the crack-tip radius is proportional to the effective energy $G=$ $2 \gamma_{\text {eff }}$ necessary to propagate the crack. We define a reference radius $a_{0}=E_{0} \gamma_{0} /\left(\pi \sigma_{c}^{2}\right)$, and obtain $a / a_{0}=G / G_{0}$.

We determine $T_{q}$ from the temperature field $T(\mathbf{x}, t)$ which satisfies the diffusion equation

$$
\frac{\partial T}{\partial t}-D \nabla^{2} T=\frac{\dot{Q}(\mathbf{x}, t)}{\rho C_{V}},
$$

where $\dot{Q}=\dot{\epsilon}_{i j} \sigma_{i j}$ is the energy production per unit volume and unit time as a result of the internal friction of the rubber. The heat diffusivity $D=\lambda / \rho C_{V}, \rho$ is the mass density, $\lambda$ the heat conductivity, and $C_{V}$ the heat capacity per unit mass. For a crack moving with constant velocity v we have $\dot{Q}(\mathbf{x}, t)=f(\mathbf{x}-\mathbf{v} t)$, so Eq. (4) can be easily solved to get $T(\mathbf{x}, t)=T_{0}+\left(\rho C_{V}\right)^{-1} \int d^{2} q(-i \mathbf{q} \cdot \mathbf{v}+$ $\left.D q^{2}\right)^{-1} f(\mathbf{q}) e^{i \mathbf{q} \cdot(\mathbf{x}-\mathbf{v} t)}$, where $f(\mathbf{q})$ is the Fourier transform of $f(\mathbf{x})$. In our study we have neglected the angular dependence of the viscoelastic energy dissipation $\dot{Q}$ so that $f(\mathbf{x})$ depends only on $r=|\mathbf{x}|$. This gives [8]

$$
\frac{T_{y}}{T_{0}}=1+\Lambda \frac{4}{\pi} \int_{0}^{1} d x x \int_{x}^{1} d z z^{-1 / 2} \operatorname{Im}\left[\frac{E_{0}}{E\left(\omega_{c} x, T_{z}\right)}\right]\left(z^{2}-x^{2}\right)^{-1 / 2} \int_{0}^{1} d w \frac{J_{0}(w / y) H(w, z)}{\left(1+\xi^{2} w^{2}\right)^{1 / 2}}
$$

where $J_{0}$ is the zero order Bessel function and

$$
H(p, q)= \begin{cases}p^{-3 / 2}\left[{ }_{2} F_{1}\left(3 / 4,3 / 4 ; 1 ; q^{2} / p^{2}\right)\right] & q<p \\ q^{-3 / 2}\left[{ }_{2} F_{1}\left(3 / 4,3 / 4 ; 1 ; p^{2} / q^{2}\right)\right] & p<q\end{cases}
$$

where ${ }_{2} F_{1}$ is the Gauss's hypergeometric function. In (5), $\xi=\left(a_{0} v_{0} / a v\right) \xi_{0}$ and $\Lambda=\gamma_{0} /\left(\rho a_{0} C_{V} T_{0}\right)$ where the dimensionless diffusivity $\xi_{0}=2 \pi D /\left(a_{0} v_{0}\right)$, and where $v_{0}$ is a reference velocity. We will assume that the WilliamsLandel-Ferry (WLF) transform [9] is valid so that $E(\omega, T)=E\left(\omega a_{T}\right)$, where $a_{T}=\exp \left[-8.86\left(T-T_{\mathrm{g}}-\right.\right.$ $\left.50) /\left(51.5+T-T_{\mathrm{g}}\right)\right]$, where $T_{\mathrm{g}}$ is the glass transition temperature. The WLF relationship is based on the thermally activated (free volume) theory of viscosity [10].

We assume that the rubber obeys the very simple Kelvin rheological model characterized by a single relaxation time $\tau$. Thus, the complex elastic modulus of the rubber is $1 / E(\omega, T)=1 / E_{\infty}+\left(1 / E_{0}-1 / E_{\infty}\right)\left(1-i \omega a_{T} \tau\right)^{-1}$. In this case the reference velocity $v_{0}=a_{0} /(2 \pi \tau)$. Within the Kelvin model, $\gamma_{\text {eff }} / \gamma_{0}$ as a function of $v / v_{0}$ depends only on the parameters $\Lambda, E_{\infty} / E_{0}, T_{0}-T_{\mathrm{g}}$, and $\xi_{0}$. Since typically $\gamma_{0} \approx 30 \mathrm{~J} / \mathrm{m}^{2}, \quad a_{0} \approx 3 \mathrm{~nm}, \quad \rho \approx 10^{3} \mathrm{~kg} / \mathrm{m}^{3}$, $C_{V} \approx 10^{3} \mathrm{~J} /(\mathrm{kg} \mathrm{K})$. and $T_{0}=20^{\circ} \mathrm{C}$ we get $\Lambda \approx 30$. In all numerical calculations presented in this Letter we have used $E_{\infty} / E_{0}=1000, T_{0}-T_{\mathrm{g}}=50 \mathrm{~K}, \Lambda=10$ or 100 , and several values for $\xi_{0}$.

Figure 1 shows the effective energy $\gamma_{\text {eff }}$ to propagate the crack as a function of the crack propagation speed for $\Lambda=$ 100 and for two different values of $\xi_{0}=2 \pi D \tau / a_{0}^{2}$ [11]. Also shown is the isothermal solution $\left(T \equiv T_{0}\right.$; dashed line). At low crack-tip velocities the influence of the flash temperature is negligible and all three curves overlap. At higher crack velocities the effective energy $\gamma_{\text {eff }}$ required to propagate the crack is nonmonotonic, exhibiting a local maximum and a local minimum, which may give rise to crack propagation instabilities. As the velocity is increased further, $\gamma_{\text {eff }}$ finally increases proportional to the crack speed. Note also that for the isothermal case, the quantity $\gamma_{\text {eff }} / \gamma_{0}$ goes asymptotically to $E_{\infty} / E_{0}$ for very high cracktip velocities [12-17].

Figure 2 shows the effective energy $\gamma_{\text {eff }}$ to propagate the crack as a function of the crack propagation speed for $\Lambda=$ 10 and for different values of $\xi_{0}$. Three different regimes

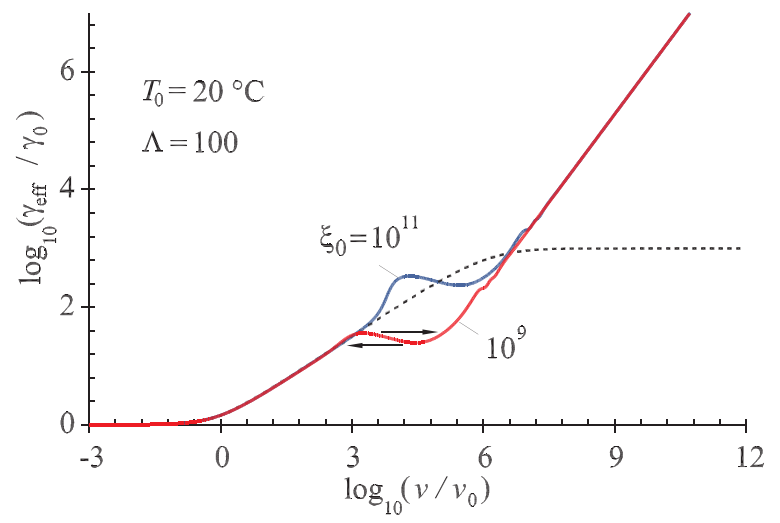

FIG. 1 (color). The effective energy $\gamma_{\text {eff }}$ to propagate the crack as a function of the crack velocity $v$ for two different values of the dimensionless diffusivity $\xi_{0}$. The dashed line is obtained by neglecting the flash-temperature effect. 


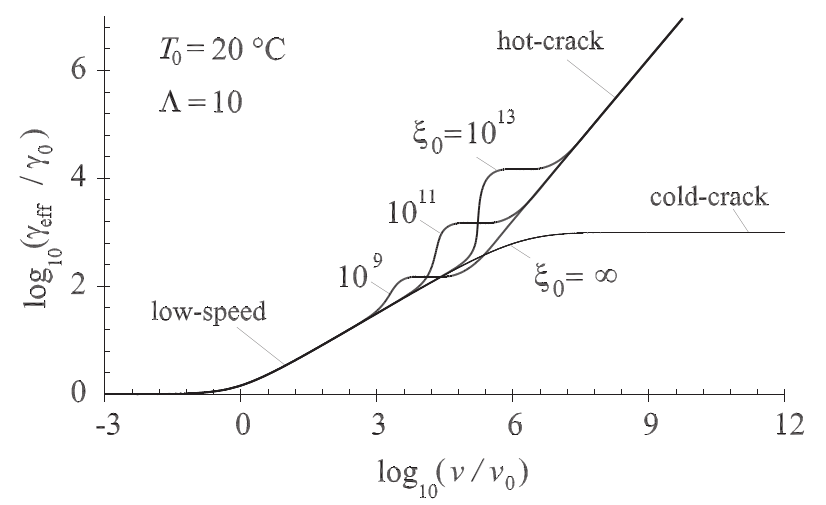

FIG. 2. The effective energy $\gamma_{\text {eff }}$ to propagate the crack as a function of the crack velocity $v$ for different values of the dimensionless diffusivity $\xi_{0}$.

are shown: (i) the low-speed regime where the increase of temperature in the rubber is negligible, (ii) the hot-crack regime, and (iii) the cold-crack regime corresponding to isothermal condition.

In Fig. 3 we show the temperature profile in the rubber, as a function of the distance from the crack tip in units of $a$, for different crack velocities. Notice that for large crack-tip velocities, the distance region in front of the crack tip where $T \gg T_{0}$ is quite large, of order $\sim 10 a$.

The reason the crack propagation energy increases (for large crack-tip velocities) when the crack-tip flashtemperature effect is taken into account can be understood as follows. Consider a propagating crack and let us first neglect the flash-temperature effect, i.e., we assume that the temperature everywhere equals the background temperature $T_{0}$ [Fig. 4(a)]. At high crack-tip velocity $v$, the region close to the crack tip is effectively in the glassy state, and contributes very little to the total viscoelastic energy dissipation. Similarly, the region very far away from the crack tip is effectively in the rubbery region of the viscoelastic spectra, and contributes also very little to the total energy dissipation. Most of the viscoelastic energy dissipation occurs in the transition region between the

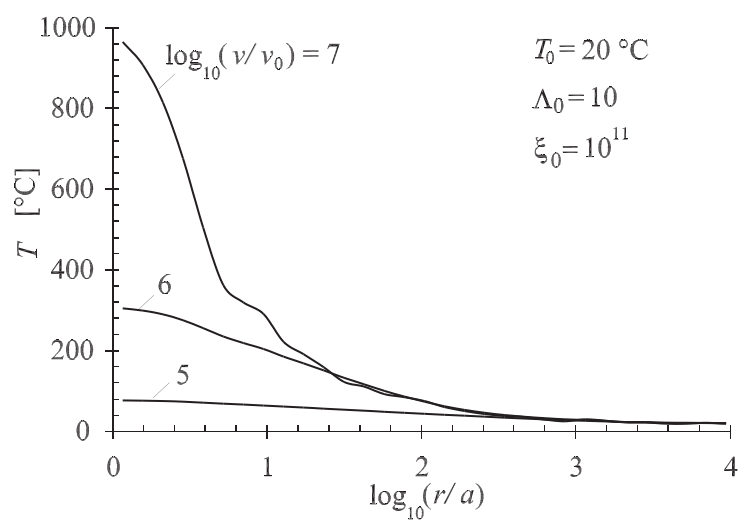

FIG. 3. The temperature profile in front of the crack tip for $\xi_{0}=10^{11}$ and for three different values of crack-tip velocity. The temperature increase is significant for $r \lesssim 100 a$. rubbery and glassy region (dark gray area in Fig. 4). Now, let us include the flash-temperature effect. Because the crack tip moves toward the region where the viscoelastic energy dissipation occurs, the temperature increase is highest close to the crack tip. Thus, part of what was the glassy region when the temperature effect was neglected will now correspond to the transition region and will contribute strongly to the total viscoelastic energy dissipation. On the other hand, what was the outer rim of the transition region when the flash-temperature effect was neglected will now be converted into the rubbery region, and will contribute very little to the total energy dissipation. However, since the temperature decreases monotonically with the distance from the crack tip, the reduction in the energy dissipation in the outer region is overcompensated by the increases in the energy dissipation in the inner region closer to the crack tip. The net effect is that the total energy dissipation increases when the flash-temperature effect is taken into account. By increasing the crack speed, the dark gray region will become larger and larger until it reaches the crack tip: when this happens the crack is in the asymptotic hot-crack regime.

The reason for the nonmonotonic behavior of the $G(v)$ relation can be understood as follows. In all cases $G(v)$ first increases and becomes larger than for the isothermal case before reaching the "flat" region. When this happens the crack-tip velocity is already high enough that the rubber at the crack edge is in the glassy region, or at least on the glassy side of the transition region. Thus, the initial increase in the temperature will shift the region close to the crack tip into the middle of the transition region of the viscoelastic spectra, and $G(v)$ increases beyond the iso-

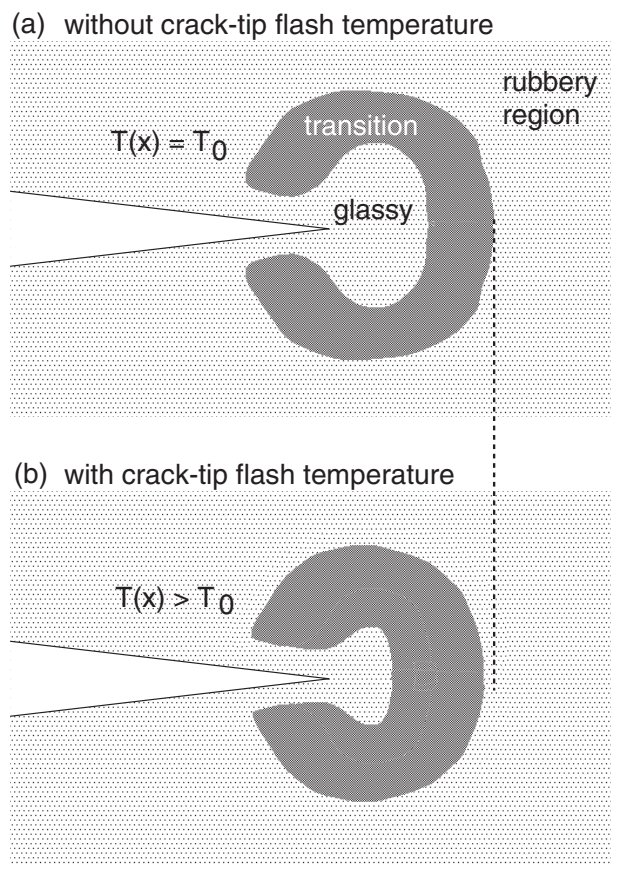

FIG. 4. A qualitative picture of what happens when the flash temperature is included in the theory. See text for details. 
thermal curve. However, a further increase in the velocity increases the temperature even more and shifts the region close to the crack tip toward the rubbery side of the transition region in such a way that $G(v)$ is now nearly constant (or even decreasing). However, at very high velocities the region close to the crack tip get shifted back towards the middle of the transition region, and then it stays there for arbitrary high crack-tip velocity; this is the so called hot-crack regime.

The theory presented above explains why unstable crack propagation is observed [18-20]. In the transition region between the low-speed regime and the hot-crack regime, a very rapid increase of crack propagation energy is first observed (see Fig. 1), followed by an almost flat region which is smoothly connected to the hot-crack regime. The overall transition region covers a velocity range of about 2 order of magnitude. Thus, in a displacement controlled test, as the system is loaded, the crack initially moves very slowly, and when it reaches the transition region its velocity is still very small $\left(v<10^{-4} \mathrm{~m} / \mathrm{s}\right)$, so that its propagation cannot be easily detected. By increasing the displacement further, a big amount of energy is stored in the solid, and when the flat area is reached the crack starts to propagate quite fast $v>10^{-2} \mathrm{~m} / \mathrm{s}$, giving rise to the observed instability. However, the present theory cannot be used to study the detailed dynamics of the stick slip as this involves nonuniform crack-tip motion.

The most detailed experimental study of stick-slip crack propagation in rubber was presented in Ref. [20]. They observed that when the (average) crack-tip velocity is in the range $\sim 0.1-10 \mathrm{~cm} / \mathrm{s}$ unstable crack propagation occur. In this velocity region cracks grow at a slow or fast rate. Furthermore, the slope of the $\log G-\log v$ curve is much higher for the high-velocity branch. Both these facts are in good qualitative agreement with our analytical results (see Figs. 1 and 2). In Ref. [20] it was also found that the crack surfaces during slow crack propagation (left branch in Fig. 2) was very rough, while very smooth crack surfaces resulted when the crack propagated fast (right branch in Fig. 2). However, this change in surface morphology is not the primary reason for the two $G(v)$ crack propagation branches, but rather a consequence of it. Thus, we believe that at high crack-tip velocities, the high temperature at the crack tip will result in a "liquidlike" region at the crack tip. This in turn will results in the formation of thin uniform layers of modified (degraded) rubber on the crack surfaces $[21,22]$. We note that it should be possible to study the temperature rise in the vicinity of the crack tip using an infrared camera with high spatial and temporal resolution.

Recent experiments [23] have detected fast crack propagation in natural rubber, where the crack-tip velocity $\sim 60 \mathrm{~m} / \mathrm{s}$ is of the order of rubber sound velocity. Crack propagation in natural rubber is more complex than for most other types of rubber because of strain crystallization close to the crack tip.
An important problem is to determine under what circumstances crack propagation will follow the hot-crack branch and the cold-crack branch. If the crack-tip velocity is increased very slowly so that the temperature field around the crack tip can be fully developed, our calculations [8] show that for all physically reasonable rubber parameters, the crack will always follow the hot-crack branch. However, if the crack tip is initially accelerating very fast, there is not enough time for the full temperature distribution to develop, and in this case the system may follow the cold-crack branch. Thus, we believe that the path the system takes depends on how the crack is generated initially.

[1] D.F. More, The Friction and Lubrication of Elastomer (Pergamon, Oxford, 1972).

[2] A. N. Gent and J. Schultz, J. Adhesion 3, 281 (1972).

[3] D. Maugis and M. Barquins, J. Phys. D 11, 1989 (1978).

[4] A. N. Gent, Langmuir 12, 4492 (1996).

[5] A. A. Griffith, Phil. Trans. R. Soc. A 221, 163 (1920).

[6] B. N. J. Persson and E. A. Brener, Phys. Rev. E 71, 036123 (2005).

[7] T.L. Anderson, Fracture Mechanics: Fundamentals and Applications (CRC Press, Boca Raton, 1995).

[8] G. Carbone and B. N. J. Persson, Eur. Phys. J. E 17, 261 (2005).

[9] M. L. Williams, R. F. Landel, and J.D. Ferry, J. Am. Chem. Soc. 77, 3701 (1955).

[10] See, e.g., U. Eisele, Introduction to Polymer Physics (Springer, Berlin, 1990).

[11] The rubber heat diffusivity $D \approx 10^{-7} \mathrm{~m}^{2} / \mathrm{s}$, and using typical values for the rubber relaxation time $\tau=0.01 \mathrm{~s}$ and for the zero-velocity crack-tip radius (which is of order the distance between two entanglements), $a_{0}=$ $3 \mathrm{~nm}$ gives $\xi_{0}=10^{9}$. However, the rubber relaxation time $\tau$ (and hence $\xi_{0}$ ) varies by many orders of magnitude between different types of rubber.

[12] P. G. de Gennes, Langmuir 12, 4497 (1996).

[13] R. M. Christensen, Int. J. Fract. 15, 3 (1979).

[14] J. A. Greenwood and K. L. Johnson, Philos. Mag. A 43, 697 (1981).

[15] R. A. Schapery, Int. J. Fract. 11, 141 (1975); 11, 369 (1975); 11, 549 (1975); 39, 163 (1989).

[16] M. Barber, J. Donley, and J. S. Langer, Phys. Rev. A 40, 366 (1989).

[17] J. M. Baney and C. Y. Hui, J. Appl. Phys. 86, 4232 (1999); C. Y. Hui, J. M. Baney, and E. J. Kramer, Langmuir 14, 6570 (1998).

[18] E. E. Gdoutos, P. M. Schubel, and I. M. Daniel, Strain 40, 119 (2004).

[19] J. T. South, S. W. Case, and K. L. Reifsnider, Mech. Mater. 34, 451 (2002).

[20] K. Tsunoda, J. J. C. Busfield, C. K. L. Davies, and A. G. Thomas, J. Mater. Sci. 35, 5187 (2000).

[21] P. G. Fox and Soria-Ruiz, Proc. R. Soc. A 317, 79 (1970).

[22] K. N. G. Fuller, P. G. Fox, and J. E. Field, Proc. R. Soc. A 341, 537 (1975).

[23] M. Marder, Phys. Rev. Lett. 94, 048001 (2005). 